\title{
A Survey of X-ray Point Sources in the Magellanic Clouds
}

\author{
A.P. Cowley ${ }^{1}$, P.C. Schmidtke ${ }^{1}$, V.A. Taylor and T.K. McGrath \\ Dept. of Physics \& Astronomy, Arizona State University, Tempe, AZ \\ 85287-1504
}

\author{
J.B. Hutchings ${ }^{1}$ and D. Crampton ${ }^{1}$ \\ Dominion Astrophysical Observatory, $H I A / N R C$, Victoria $B C$, \\ V8X $4 M 6$ Canada
}

\begin{abstract}
.
In this study we compare the global populations of stellar X-ray sources in the LMC, SMC, and the Galaxy. After removing foreground stars and background AGN from the samples, the relative numbers of the various types of X-ray point sources within the LMC and SMC are similar, but differ markedly from those in the Galaxy. The Magellanic Clouds are rich in high-mass X-ray binaries (HMXB), especially those containing rapidly rotating Be stars. However, the LMC and SMC both lack the large number of low-mass X-ray binaries (LMXB) found in the Milky Way, which are known to represent a very old stellar population based on their kinematics, chemical composition, and spatial distribution.
\end{abstract}

\section{Introduction}

X-ray surveys of the LMC and SMC were carried out using Einstein Observatory (Long et al. 1981, Wang et al. 1991, Seward \& Mitchell 1981, Wang \& Wu 1992). These revealed several hundred point sources with $\mathrm{L}_{X} \geq 10^{35} \mathrm{erg} \mathrm{s}^{-1}$ in the direction of the Magellanic Clouds, but many were unidentified because of the relatively large positional uncertainties and the optically crowded fields in these galaxies (see Cowley et al. 1984).

In an effort understand the global populations of the X-ray sources in the Magellanic Clouds, it was necesssary to identify the remaining sources. Thus, we undertook an observational program using the ROSAT High Resolution Imager (HRI). We observed all previously known but unidentified Einstein point sources. For those detected by $R O S A T$, we then measured accurate positions $\left( \pm 5^{\prime \prime}\right)$ and fluxes. A high percentage of these sources were found to be variable in X-rays. In addition, of the previously known Einstein sources, 49 in the LMC and 36 in the SMC were not detected with $R O S A T$, although their catalogued fluxes were well above the detection threshold. This indicates that either they are highly

\footnotetext{
${ }^{1}$ Visiting Astronomers, Cerro Tololo Inter-American Observatory. CTIO is operated by AURA, Inc. under cooperative agreement with the National Science Foundation
} 
variable (transients) or spurious detections in the Einstein searches. In addition, new sources were often detected in the $R O S A T$ images, and these were included in our study.

\section{Observations and Results}

Using photometry and spectroscopy obtained at CTIO, we have identified nearly all the ROSAT-detected sources (Schmidtke et al. 1994, Cowley et al. 1997, Schmidtke et al. 1999). Many of these sources are found to be foreground stars or background AGN, leaving a very small number of actual Magellanic Cloud members. We find that the spatial distribution of optically identified point sources is widely spread over both the Clouds, with a radius of $\sim 8^{\circ}$ from the dynamical center of the LMC and $\sim 3^{\circ}$ in the SMC. In the LMC there is no obvious concentration to the star-rich Bar.

In the histograms in Fig. 1 we compare the number of various types of $\mathrm{X}$-ray point sources found the Galaxy, the LMC, and the SMC. The data for the galactic sources are taken from the catalogue of van Paradijs (1995). We have divided the data into several groups. The massive X-ray binaries (MXRB) include systems with supergiant primaries (s.g.) and the longer-period systems with Be-star primaries (Be), many of which contain slow X-ray pulsars. The Be-star systems are especially common in the Magellanic Clouds and may tell us something about the rapid rotation of young stars in these galaxies. Because all of the MXRB contain massive early-type stars, we know they are relatively young objects.

In contrast, both the LMC and SMC lack the numerous low-mass X-ray binaries (LMXB) which dominate the X-ray source population in the Milky Way. In the Galaxy the LMXB are known to be part of an old stellar population from their kinematics, chemical compositions, and spatial distribution. The Magellanic Clouds are very deficient in the old source population represented by these LMXB. In the histograms, the LMXB are divided into two groups - those in the field (which include bursting sources, X-ray pulsars, transients, and persistent sources) and those in globular clusters. In the Magellanic Clouds, the only true LMXB is in the field (LMC X-2), and none is present in the clusters. However, since there are only about a dozen old clusters in these galaxies, perhaps the absence of X-ray sources in them is not unexpected. We stress that in both the LMC and SMC our X-ray detection limit is well below the luminosity of all the galactic X-ray binaries, both high and low mass. Many of the LMXB in the Galaxy are optically unidentified (obscured by dust in the galactic plane), but their X-ray properties make clear their source types.

Although a relatively large number of supersoft X-ray sources (SSS) are found in the Magellanic Clouds, this appears to be a selection effect rather than a true population difference. In the Galaxy these sources are highly absorbed by interstellar hydrogen gas, so they are only detected in small numbers. The lower column density of gas towards the LMC and SMC makes them more easily detected there. We note that two SSS in the LMC and one in the SMC lack optical identifications. 


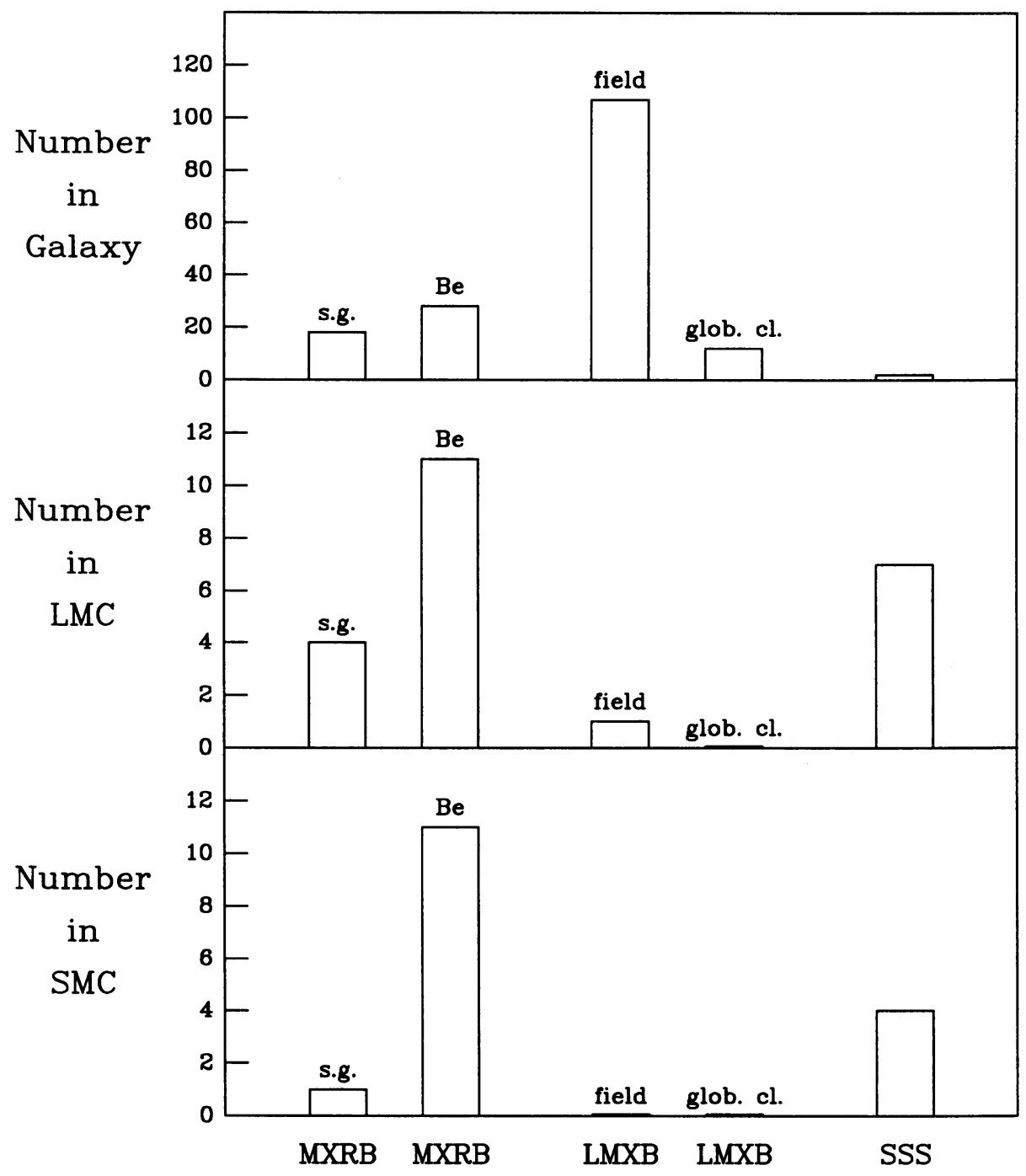

Figure 1. Comparison of the number of various types of X-ray point sources in the Galaxy, LMC, and SMC. The histograms for the Galaxy and the LMC are scaled approximately by their mass ratio (10:1). The massive X-ray binaries (MXRB) include those with early-type supergiant primaries (s.g.) and those with Be-star primaries (Be). They are relatively young objects. The old, low-mass X-ray binaries (LMXB) are divided into objects in the field and those in globular clusters. The supersoft X-ray sources (SSS) are also shown, but their numbers in the Galaxy are affected by selection effects (see text). 


\section{Ongoing and Future Work}

In a related program we have examined the much deeper $\mathrm{X}$-ray images from a ROS AT-HRI survey of the LMC being carried out by Chu et al. (1996). These observations reach to much lower flux limits $\left(\sim 10^{34} \mathrm{erg} \mathrm{s}^{-1}\right.$ in the LMC) than previous surveys, but only a portion of the LMC has so far been observed. We have created a preliminary point-source catalogue, and an optical identification program is underway.

Acknowledgements. We thank the staff at CTIO for their assistance. This work was supported in part by grants to ASU from NSF and NASA.

\section{References}

Chu, Y.-H., Snowden, S.L., Chang, T. 1996, The Rosat Newsletter, 13, 26

Cowley, A.P., Crampton, D., Hutchings, J.B., Helfand, D.J., Hamilton, T.T., Thorstensen, J.R., Charles, P.A. 1984, ApJ, 286, 196

Cowley, A.P., Schmidtke, P.C., McGrath, T.K., Ponder, A.L., Fertig, M.R., Hutchings, J.B., Crampton, D. 1997, PASP, 109, 21

Long, K.S., Helfand, D.J., Grabelsky, D.A. 1981, ApJ, 248, 925

Schmidtke, P.C., Cowley, A.P., Crane, J.D., Taylor, V.A., McGrath, T.K., Hutchings, J.B., Crampton, D. 1999, in preparation

Schmidtke, P.C., Cowley, A.P., Frattare, L.M., McGrath, T.K., Hutchings, J.B., Crampton, D. 1994, PASP, 106, 843

Seward, F.D., Mitchell, M. 1981, ApJ, 243, 736

van Paradijs, J. 1995, in: X-ray Binaries, (eds.) W.H.G. Lewin, J. van Paradijs \& E.P.J. van den Heuvel, Cambridge University Press: Cambridge, p. 536

Wang, Q., Hamilton, T., Helfand, D.J., Wu, X. 1991, ApJ, 374, 475

Wang, Q., Wu, X. 1992, ApJS, 78, 391

\section{Discussion}

Feast: Is there any possibility that the low frequency of old X-ray sources in the Magellanic Clouds is due to an underestimate of the luminosity of such sources?

Cowley: In the Galaxy, their X-ray luminosities are well established (many lie near the galactic center), $L_{X}=10^{36}$ to $10^{38} \mathrm{erg} \mathrm{s}^{-1}$. The X-ray surveys in the Magellanic Clouds reach well below this $L_{X}<10^{35} \mathrm{erg} \mathrm{s}^{-1}$ so we should have easily detected them. The one low-mass X-ray binary in the LMC (LMC X-2) has an X-ray luminosity comparable to those in the Galaxy, so they seem to be truly deficient in the LMC and SMC. 